\title{
Space Teleoperation Visual Recognition System Using A Novel Pattern Recognition Technique
}

\author{
Mingming Wang \\ Mechanical School of Engineering and Science, Huazhong University of Science and Technology. Wuhan 430074 China
}

\begin{abstract}
A Space Teleoperation Visual Recognition System is developed. It is used to recognize an object and measure the 3D pose. A highly efficient no iterative approach is used to calculator the external parameters in camera calibration. It divides all reference points into 3-point subsets in order to achieve a series of fourth order polynomials. The optimal solution is determined by solving the roots of the derivative of the cost function. Laser tracker is used to measure accurately the coordinate system of the robot, the end of the robot arm and the 3D target, then the hand-eye relation matrix is calculated based on these measurement results and external camera parameters. Then, we propose a novel pattern recognition technique to show how to reduce the dimensionality by utilizing group and convexity model. The performance of this visual system is tested in a space robot prototype.
\end{abstract}

Keywords-recognition; teleoperation; hand-eye calibration; camera calibration

\section{INTRODUCTION}

Vision system for specific space application can only use monocular vision because of limitations like volume, mass, power, field of view, etc. In addition to observing the operating scene, the camera installed on the space manipulator can also perform the measurement of the 3D pose of the target by installing $3 \mathrm{D}$ target on the adapter. According to the given relative pose between camera and adapter, we can acquire the pose of the end effector relative to the adapter or the world coordinate system of the robot arm. This vision system can automatically provide precise pose data to assist operators in completing more precise manipulation tasks.

In general, 2D calibration target has high accuracy [1] [2], and it's easy to obtain high-quality images of the target and high calibration accuracy. But the calibration accuracy turns out to be low and calibration methods are more cumbersome when the target is relatively small. 3D target[3][4] discussed here which is for space-oriented applications, have higher machining precision and calibration accuracy, and the $3 \mathrm{D}$ target calibration algorithm is easy to implement, Therefore, $3 \mathrm{D}$ target is used to achieve camera calibration in this paper.

Vision measurement refers to using only one visual sensor to capture images, and then measure objects' geometric size, pose, etc. The vision measurement system is not restricted by the field of view, the pose of the target can be measured in the desired range and distance via changing the lens. The safety of the robotic arm is threatened by non-cooperative irregular targets in the space. The camera on the robot can not only measure the pre-designed target, but also can identify the irregular objects in space. In the recognition problem the data images are translated and scaled into new values of fixed size. This method reduces the variations in the data in each dimension because the localities of all the values are same. The data sets are large in traditional sets but the increased dimension creates a problem in accuracy. The proposed research work have extracted and analyzed the pattern recognition paradigm using the group and convex model. The objective of the technique ensures the efficient recognition with reduced data sets without compromising in error factor and computation time.

In this paper, vision measurement system consists of three aspects: 1) calibration of camera internal parameters and handeye relationship, 2) calculation of the camera external parameters, and 3) identify the irregular objects in space

\section{CAMERA AND HAND-EYE RELATIONSHIP CALIBRATION}

Calibration of camera and the hand-eye relationship are arranged on the ground. The end of the robot arm moves to three different specified positions. Their 3D poses are measured accurately via a laser tracker. The internal parameter and external matrixes of the camera can be calculated firstly with the 3D coordinates and 2D image coordinates of these 14 feature points in the target. Then the transformation matrix between the camera and the end of the robot arm can be calculated with this $3 \mathrm{D}$ pose information of the arm end and the external parameters of the camera.

\section{A. Calibration of Camera Parameters}

The calibration of camera parameters focuses on determining the internal parameters and the $3 \mathrm{D}$ pose of the camera frame relative to a certain world coordinate system.

Internal camera parameters refer to the basic parameters of the camera imaging, such as the main point (image center), focal length, lens distortion and other system errors parameters. And external parameters describe the transformation from target to camera coordinate system.

The camera calibration of internal and external parameters is to establish mapping from marker (target object) coordinates to a $2 \mathrm{D}$ camera image (pixel) coordinates. This relationship is necessary for the target object pose measurement, and therefore camera calibration must be carried out. Moreover, hand-eye relationship calibration is also performed basing on camera calibration. 
Camera calibration is the process of determining the geometric and optical characteristics of a camera, namely internal parameters matrix $\mathrm{M} 1$, distortion parameters, and external parameters matrix $\left[r_{1}, r_{2}, r_{2}, t\right]$. The accuracy of camera calibration would directly affect the accuracy of the visual measurement.

The projection of feature points from target to the camera image plane could be represented by the following formula:

$$
Z_{v}\left[\begin{array}{l}
u_{1} \\
v \\
1
\end{array}\right]=M_{1}\left[r_{1}, r_{2}, r_{3}, t\right]\left[\begin{array}{c}
X_{w} \\
Y_{w} \\
Z_{w} \\
1
\end{array}\right] \text {. }
$$

Where $M_{1}$ is the camera internal parameters matrix, $(u, v, 1)$ is the pixel coordinate of feature points, and $\left(X_{w}, Y_{w}, Z_{w} 1\right)$ is the coordinate of feature points in target coordinate system.

Let M2 $=\left[r_{1}, r_{2}, r_{2}, \mathrm{t}\right]$, where M2 is external camera parameters matrix. $\left[r_{1}, r_{2}, r_{3}\right]$ is a 3 by 3 orthonormal rotation matrix, $\mathrm{t}=\left[t_{\mathrm{w},}, t_{\mathrm{y},}, t_{\mathrm{z}}\right]$ describes the translation from the target to camera coordinate system.

Let

$$
\mathrm{M}=M_{1} M_{2}=\left[\begin{array}{l}
m_{11} m_{12} m_{13} m_{14} \\
m_{21} m_{22} m_{23} m_{24} \\
m_{31} m_{32} m_{33} m_{34}
\end{array}\right] .
$$

Substitute equation (2) into equation (1), so

$$
Z_{v}=\left[\begin{array}{l}
u \\
v \\
1
\end{array}\right]=M\left[\begin{array}{c}
X_{W} \\
Y_{W} \\
Z_{W} \\
1
\end{array}\right] .
$$

For each feature point $\left(X_{w i}, Y_{w i}, Z_{w i}\right)$, their corresponding image coordinates are $\left(u_{i}, v_{i}\right)$.

According to Ji Qiang and Costa's algorithm [5], solving matrix $M$ would obtain the value of each element in $M_{1}$.

\section{B. Hand-Eye Relationship Calibration}

Let $\mathrm{X}=\left[\boldsymbol{R}_{\varepsilon} t_{\varepsilon}^{c}\right]$, where $X$ is the transformation from camera to the arm end, $R_{\varepsilon}^{c}$ is a 3 by 3 orthonormal rotation matrix, $t_{\varepsilon}^{c}$ describes the translation vector from camera to the arm end coordinate system. Considering that relative position of camera and actuator remains unchanged when the end of robot arm moves to various positions, we can solve $\mathrm{X}$ on the basis of calibrating external parameters of the camera and measuring 3D position and posture of the arm end, as shown in Fig. 1.

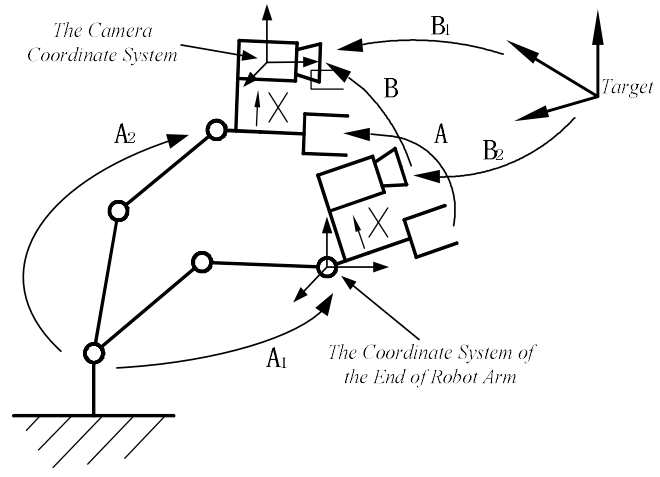

FIGURE I. SCHEMATIC ILLUSTRATION OF HAND-EYE RELATIONSHIP CALIBRATION

In Figure 1, $A$ is the relative transformation matrix between the two corresponding positions in the end of robot arm, $B$ is the relative transformation matrix between the two different positions in the camera coordinate system, then:

$$
A X=X B
$$

So it turns to solve $\mathrm{AX}=\mathrm{XB}$ for hand-eye calibration of the robot arm.

Let A1 be the initial pose matrix at the end of the robot arm, and it becomes A2 when the robot arm moves. The external parameters matrixes of the camera in the two locations are B1 and B2 respectively. Substitutes them into equation (4), so:

$$
A_{1} X B_{1}=A_{2} X B_{2}
$$

In the experiment of hand-eye calibration, $A_{1}, A_{2}, B_{1}$ and $B_{2}$ can all be accurately measured with a laser tracker, and then $X$ can be calculated by the method of matrix direct product.

\section{STYLING DETERMINATION OF THE 3D POSE OF THE OBJECT}

The key to determine the $3 \mathrm{D}$ pose of the object is the calibration of external camera parameters matrix. An efficient and noniterative RPnP method is used to calculate the external camera parameters matrix. The method can retrieve the optimum by solving a seventh order polynomial. The main idea is that it divides the reference point into 3-point subsets in order to achieve a series of fourth order polynomials, and then compute the sum of the square of the polynomials so as to form a cost function, find the roots of the derivative of the cost function in order to determine the optimum. The algorithm can stably handle the planar case, ordinary 3D case, and quasisingular case, and it is as accurate as the state-of the-art iterative algorithms with much less computational time. It is the first noniterative PnP solution that can achieve more accurate results than the iterative algorithms when no redundant reference points can be used $(\mathrm{n} \leq 5)$. Large-size point sets can be handled efficiently because its computational complexity is $O(n)$. The detailed introduction to the RPnP algorithm can be found in [6].

In practice, we estimate the camera pose from each local minimum and select the result with the least reprojection residual as the optimum of the solution, and then the rotation 


\section{REFERENCES}

[1] Z. Zhang, "A flexible new technique for camera calibration," Pattern Analysis and Machine Intelligence, IEEE Transactions on 22.11 (2000): 1330-1334.

[2] R. Y. Tsai, "A versatile camera calibration technique for high-accuracy 3D machine vision metrology using off-the-shelf TV cameras and lenses," Robotics and Automation, IEEE Journal of 3.4 (1987): 323-344.

[3] J. Heikkila, O. Silvén, "A four-step camera calibration procedure with implicit image correction," Computer Vision and Pattern Recognition, 1997. Proceedings., 1997 IEEE Computer Society Conference on. IEEE, 1997.

[4] P. F. Sturm, S. J. Maybank, "On plane-based camera calibration: A general algorithm, singularities, applications," Computer Vision and Pattern Recognition, 1999. IEEE Computer Society Conference on. Vol. 1. IEEE, 1999.

[5] Q. Ji, M. S. Costa, R. M. Haralick, L. G. Shapiro, "An integrated linear technique for pose estimation from different geometric features," International Journal of Pattern Recognition and Artificial Intelligence, 1999, 13(05): 705-733.

[6] S. Li, C. Xu*, M. Xie, "A Robust O(n) Solution to the Perspective-nPoint Problem," IEEE Transactions on Pattern Analysis and Machine Intelligence, 2012. 